\title{
Effect of Fe Content on Atomic and Electronic Structure of Complex Oxides $\mathrm{Sr}(\mathrm{Ti}, \mathrm{Fe}) \mathrm{O}_{3-\delta}$
}

Elena O. Filatova ${ }^{*}$, Yulia V. Egorova ${ }^{1}$, Kristina A. Galdina ${ }^{1}$, Tobias Scherb ${ }^{2}$, Gerhard Schumacher ${ }^{2}$, Henny J.M. Bouwmeester ${ }^{3}$, Stefan Baumann ${ }^{4}$

${ }^{1}$ Institute of Physics, St-Petersburg State University, Ulyanovskaya Str. 3, Peterhof 198504, St. Petersburg, Russia

${ }^{2}$ Helmholtz-Zentrum Berlin für Materialien und Energie GmbH, Hahn-Meitner-Platz 1, D-14109 Berlin, Germany

${ }^{3}$ University of Twente, P.O. Box 217, 7500 AE Enschede, The Netherlands

${ }^{4}$ Forschungszentrum Juelich, Wilhelm-Johnen-Straße, 52425 Juelich, Germany

Email: feo@ef14131.spb.edu

Phone: +7 (812) 4284352 


\begin{abstract}
We present a study of the electronic and atomic structure of two series of $\mathrm{SrTi}_{1-\mathrm{x}} \mathrm{Fe}_{\mathrm{x}} \mathrm{O}_{3-\delta}$ (STFO) powders with different Fe content produced by two different methods, spray pyrolysis or modified Pechini synthesis, by means of soft X-ray absorption spectroscopy. Partial substitution of Ti by Fe atoms in $\mathrm{SrTiO}_{3}$ were found to cause asymmetric distortion of $\mathrm{TiO}_{6}$ octahedrons which increases with increasing Fe content that may violate the cubic symmetry of STFO. The presence mainly of $\mathrm{Fe}^{3+}$ states in octahedral environment at small concentration of $\mathrm{Fe}$ atoms along with essentially smaller content of $\mathrm{Fe}^{4+}$ states in octahedral environment where the latter contribution increases with increasing Fe content was traced. It is found that the modified Pechini method allows to synthesize more stable structures but a tendency of the $\mathrm{SrO}_{x}$ formation in the structure prepared by this technique was marked. The spray pyrolysis method gives the structure free of SrO precipitates but the presence of $\mathrm{Fe}^{3+}$ states in tetrahedral environment with $\mathrm{Fe}$ content higher than $50 \%$ and even a certain amount of $\mathrm{Fe}^{2+}$ ions in an octahedral environment at concentrations higher than $75 \%$ in the STFO prepared by this method was established. The O1s (K)- absorption spectra point to increase in oxygen vacancy concentration with increasing Fe content. The lowest degree of structure distortions with enough high oxygen vacancy concentration was traced in STFO $(x=0.25$ to $x=0.35)$ produced by modified Pechini synthesik, which makes it mostly appropriate for technical applications, e.g., as gas sensors, oxygen separation membranes or as fuel cell material.
\end{abstract}




\section{Introduction}

Nowadays, solid oxide fuel cell (SOFC) cathodes are almost exclusively fabricated using perovskite type materials [1]. For a long time ( $\mathrm{La}, \mathrm{Sr}) \mathrm{MnO}_{3}$ cathodes have gained broad popularity [2]. However, their main drawback is related to the primarily electronic nature of conductivity and high activation energy for oxygen reduction reaction [3, 4]. Among other criteria, SOFC cathode materials should reveal good ionic conductivity to form a mixed conducting electrode, which can provide higher electrochemical performance. One possible candidate is perovskite of the strontium titanate (STO) group. Due to its good dielectric properties, STO has been extensively studied. $\mathrm{SrTiO}_{3}$ is essentially a wide band gap semiconductor with low conductivity $[5,6]$ and is stable at high temperatures. STO can be either acceptor or donor doped that allows us to vary its properties for many different applications. As follows from [7] acceptor doping, e.g. partial substitution of titanium by iron results in high oxygen conductivity due to a charge compensation by appearance of oxygen vacancies, whereas in donor-doped STO the bulk conductivity is controlled by metal vacancies and therefore exhibits a long-term drift behavior due to cation diffusion.

Recently, strontium titanium ferrite $\mathrm{SrTi}_{1-\mathrm{x}} \mathrm{Fe}_{\mathrm{x}} \mathrm{O}_{3-\delta}(\mathrm{STFO})$ solid solution has attracted much attention because these materials exhibit a p-type conducting behavior at high temperatures and may thus be applied in resistive gas sensor devices for oxygen and hydrocarbons [8-11]. It can also be used as cathodes for SOFC [12] and as oxygen separation membranes [13].

The $\mathrm{SrTi}_{1-\mathrm{x}} \mathrm{Fe}_{\mathrm{x}} \mathrm{O}_{3-\delta}$ sequence forms a continuous solid solution between the end members $\mathrm{SrTiO}_{3}$ and $\mathrm{SrFeO}_{3}$. Pure $\mathrm{SrFeO}_{3-\delta}$ undergoes an order-disorder phase transition below $850^{\circ} \mathrm{C}$ from disordered perovskite to ordered brownmillerite $\left(\mathrm{Sr}_{2} \mathrm{Fe}_{2} \mathrm{O}_{5}\right)$, which limits its application $[14,15]$. Notice that the ionic and electronic conductivities change over several orders of magnitude by partial substitution of titanium by iron in $\mathrm{SrTiO}_{3}$. The substitution of iron by titanium in $\mathrm{SrFeO}_{3}$ stabilizes the disordered perovskite phase retaining a high electronic and ionic conductivity over a wide range of oxygen partial pressures and temperatures [15, 16]. Therefore, it is necessary to find the optimal chemical composition of $\mathrm{SrTi}_{1-\mathrm{x}} \mathrm{Fe}_{\mathrm{x}} \mathrm{O}_{3-\delta}$ solid solution that possesses a stable cubic perovskite phase while maintaining the high electronic and ionic conductivity. Jung and Tuller [17-19] found that a resistivity of $\mathrm{SrTi}_{1-\mathrm{x}} \mathrm{Fe}_{\mathrm{x}} \mathrm{O}_{3-\delta}$ with $\mathrm{x}=0.35$ and 0.5 is significantly lower than resistivity for the typical SOFC electrodes, that certainly makes this composition very important for the structural, atomic and electronic analysis.

The current work focuses on the changes in atomic and electronic structure of $\operatorname{SrTi}_{1-\mathrm{x}} \mathrm{Fe}_{\mathrm{x}} \mathrm{O}_{3-\delta}(0.25 \leq \mathrm{x} \leq$ 0.90\%) upon Fe substitution on the transition metal site produced by two different methods, spray pyrolysis or modified Pechini synthesis.

All the studies were carried out using near edge $\mathrm{x}$-ray absorption fine structure (NEXAFS) spectroscopy. Fine structure arising in the vicinity of absorption edges provides information about local 
(associated with a hole localization in the core shell) and partial (allowed for certain angular momentum symmetry) electronic density of states of the conduction band. NEXAFS is dominated by multiple scattering of low-energy photoelectrons in the valence potential set up by the nearest neighbours that allows us to use a spectral "fingerprint" technique to identify the local bonding environment that defines the highest sensitivity of NEXAFS to distinguish chemical bonds and local coordination.

\section{Experimental Methods}

$\mathrm{SrTi}_{1-\mathrm{x}} \mathrm{Fe}_{\mathrm{x}} \mathrm{O}_{3-\delta}$ powders with partial Fe substitution for Ti cations $(\mathrm{x}=0.3,0.5,0.7,0.9)$ were prepared by spray pyrolysis (Norwegian University of Science and Technology (NTNU), Norway) in a continuous air flow at $860{ }^{\circ} \mathrm{C}$, using precursor solutions containing stoichiometric amounts of cations. The obtained raw powders were calcined at $950{ }^{\circ} \mathrm{C}$ for $12 \mathrm{~h}$ in stagnant air and ball-milled in ethanol for $24 \mathrm{~h}$ [20]. The phase purity was checked using X-ray powder diffraction (D2 PHASER, Bruker AXS, Germany) with Cu Ka. A continuous scan mode was used to collect diffraction date in the range $2 \theta_{-}=20-90^{\circ}$ with a step size of $0.02^{\circ}$ and counting time of 1 s/step.

A second series of $\mathrm{SrTi}_{1-\mathrm{x}} \mathrm{Fe}_{\mathrm{x}} \mathrm{O}_{3-\delta}$ powders with $\mathrm{x}=0.25,0.35,0.5 .0 .75$ was produced by a modified Pechini synthesis [21]. The obtained raw powders were calcined at $1100{ }^{\circ} \mathrm{C}$ for $5 \mathrm{~h}$ in stagnant air and ball-milled in ethanol for $24 \mathrm{~h}$. The NEXAFS measurements were performed at the RGL-station on the Russian-German beamline (RGBL) at the BESSY II synchrotron light source of the Helmholtz-Zentrum Berlin [22]. The spectra were measured at the incident angle of $45^{\circ}$ in the vicinity of $\mathrm{Fe} \mathrm{L}_{2,3}$, $\mathrm{Ti} \mathrm{L}_{2,3}$ and $\mathrm{O} \mathrm{K}$-absorption edges with energy resolution better than $\mathrm{E} / \Delta \mathrm{E}=2000$. The spectra were obtained by monitoring $\theta$ f the total electron yield (TEY) from the samples in the current mode. The absolute energy calibration was carried out by measuring $\phi f$ the energies of the reference $\mathrm{Au} 4 \mathrm{f}$ 7/2 photoelectron peak $(83.95 \mathrm{eV})$ in different diffraction orders.

\section{Results and Discussion}

\subsection{Titanium 2p X-ray absorption}

The Ti 2p $\left(\mathrm{L}_{2,3}\right)$ - absorption spectra of $\mathrm{SrTi}_{1-\mathrm{x}} \mathrm{Fe}_{\mathrm{x}} \mathrm{O}_{3-\delta}(\mathrm{STFO})$ powders with different $\mathrm{Fe} / \mathrm{Ti}$ ratio for both of series of STFO samples are plotted in Figure 1. The $\mathrm{Ti} \mathrm{L}_{2,3}$ - absorption spectrum of $\mathrm{SrTiO}_{3}$ measured under the same experimental conditions is also shown in Figure 1. 
a)

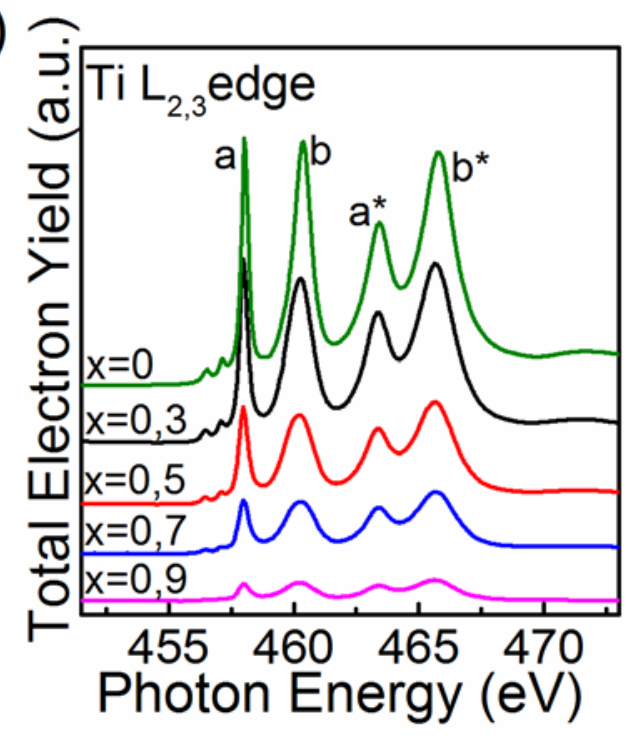

b)

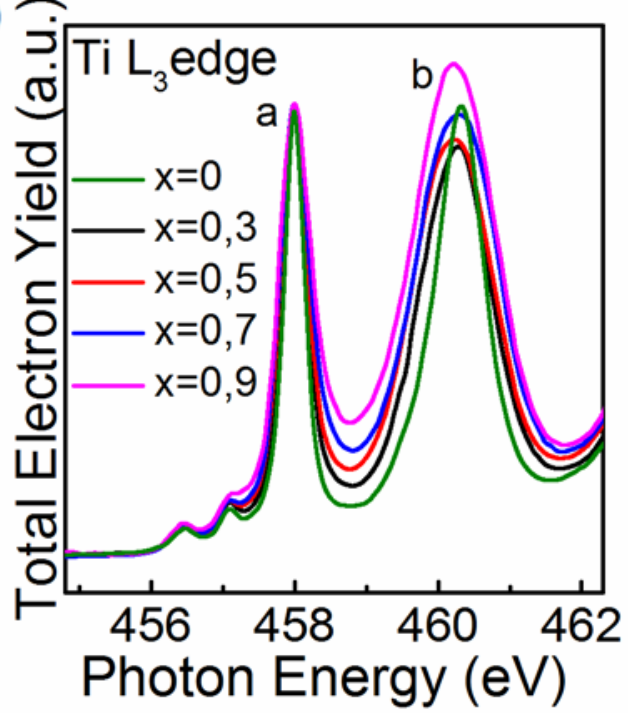

c)

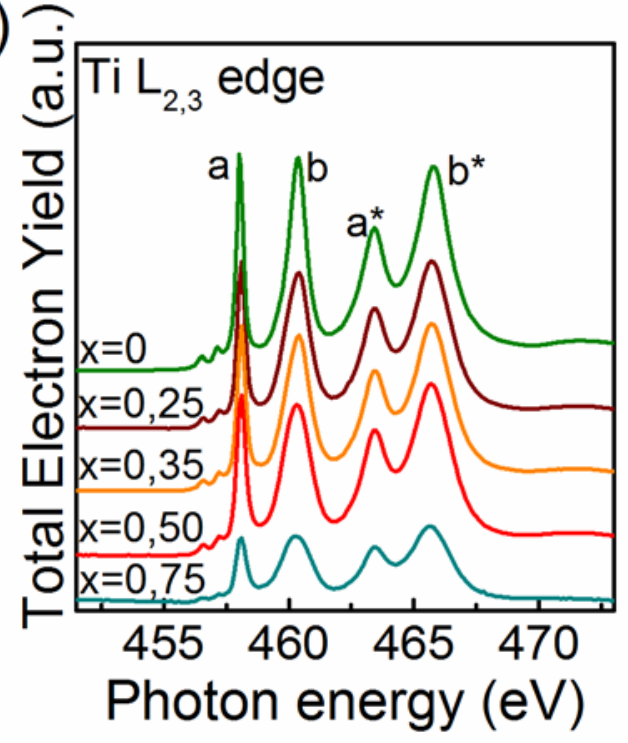

d)

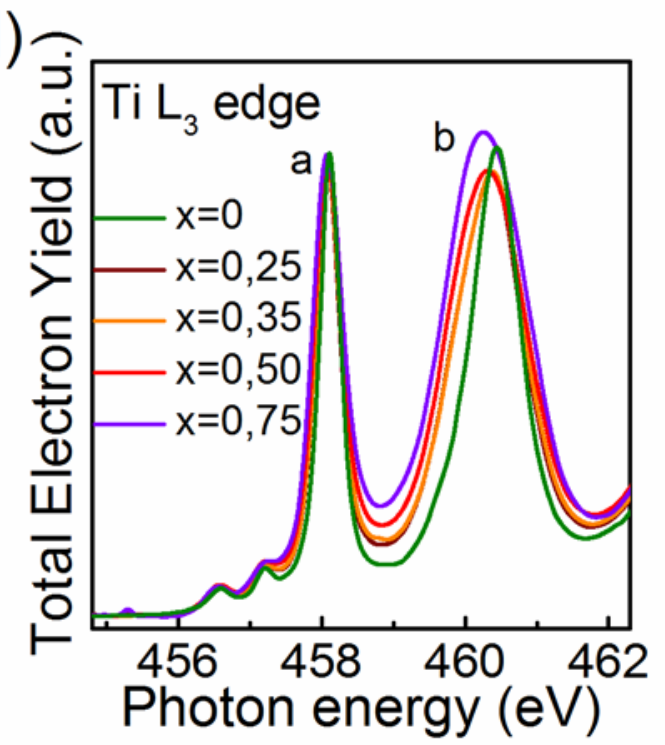

Figure 1. Ti2p ( $\left.\mathrm{L}_{2,3}\right)$ - absorption spectra of the $\mathrm{SrTi}_{1-\mathrm{x}} \mathrm{Fe}_{\mathrm{x}} \mathrm{O}_{3-\delta}$ (STFO) with different content of substituted Fe atoms and $\mathrm{SrTiO}_{3}$ powders. (a-b) a group of samples with x: 0.3, 0.5, 0.7 and 0.9 at of Fe on Ti site produced by spray pyrolysis method. (c-d) a group of samples with $\mathrm{x}$ : 0.25, 0.35, 0.50 and 0.75 of Fe on Ti site produced by modified Pechini synthesis. Panels (b) and (d) show the Ti 2 $\mathrm{p}_{3 / 2}\left(\mathrm{~L}_{3}\right)$ absorption spectra normalized on the intensity of feature a. The $\mathrm{Ti} \mathrm{L}_{2,3}$ - absorption spectrum of $\mathrm{SrTiO}_{3}$ measured under the same experimental conditions is also shown.

The ideal cubic $\mathrm{SrTiO}_{3}$ can be considered as a structure constructed from the (TiO6) ${ }^{8-}$ clusters. In a simple ionic model, $\mathrm{Ti}$ in $\mathrm{SrTiO}_{3}$ is tetravalent and has no 3d electrons. The bottom of the conduction band is formed by the Ti 3d states, which are strongly mixed with the oxygen states. This leads to the situation in which 
non-vanishing 3d electrons exist in the ground state [23-27]. The empty and antibonding Ti 3d band is split under the field of surrounding oxygen atoms into $t_{2 g}$ ( $\pi$ bonds) and $e_{g}$ ( $\sigma$ bonds) sub-bands.

According to the classical conception, the NEXAFS excitation at the Ti $2 p$ threshold should reflect the energies of the empty Ti $3 \mathrm{~d}$ states, because it is dominated by the $2 \mathrm{p} \rightarrow 3 \mathrm{~d}$ dipole transitions in the Ti atoms. One can see that the Ti 2p absorption spectra of both series (Figure 1a, c) reflect clearly the spin-orbit splitting of the $\mathrm{Ti} 2 \mathrm{p}$ level. The Ti 2p1/2 ( $\mathrm{L}_{2}$-feature) structures are marked by asterisks in Figure 1 (a, c). The $\mathrm{L}_{2}$ absorption spectra are characterized by a broad structure that is due to an additional damping channel caused by the $\mathrm{L}_{2} \mathrm{~L}_{3} \mathrm{~V}$ - Costere - Kronig transition [28], which reduces the life time of $\mathrm{L}_{2}$-holes. Therefore, we will focus on evolution of the $2 \mathrm{p}_{3 / 2}$-absorption spectra. The peaks $\boldsymbol{a}$ and $\boldsymbol{b}$ in the Ti $2 \mathrm{p}_{3 / 2}$ spectrum stem from the dipole allowed transitions of the Ti 2 $\mathrm{p}_{3 / 2}$ electrons to unoccupied 3d states split into $3 \mathrm{dt}_{2 \mathrm{~g}}$ (peak $\boldsymbol{a}$ ) and 3deg (peak $\boldsymbol{b}$ ) components by the octahedral crystal field created mainly by the oxygen ions.

A joint analysis of the Ti 2p-absorption spectra for all the studied samples reveals that the spectra are very similar in shape and energy position of their main features but differ in their intensity (Figure1a,c). The intensity of all the features of the Ti 2p-absorption spectra of STFO decreases with increasing iron content. Besides, the dependence of the width of the peaks $\boldsymbol{a}$ and $\boldsymbol{b}$ (most explicitly of $\boldsymbol{b}$ ) on the content of Fe atoms is traced. Panels (c) and (d) in Figure 1 show the Ti 2p $3 / 2\left(\mathrm{~L}_{3}\right)$ absorption spectra normalized on the intensity of feature $\boldsymbol{a}$.

Recall that ideal cubic $\mathrm{SrTiO}_{3}$ is characterized by a structure with undistorted and unrotated $\mathrm{TiO}_{6}$ octahedra. The violation of the cubic symmetry due to the asymmetric distortion of $\mathrm{TiO}_{6}$ octahedra leads to modifications in shape of the absorption spectrum. Calculations, carried out in ref. [29], revealed that lowering of the symmetry from cubic to tetragonal or trigonal symmetry leads to the broadening of the features $\boldsymbol{a}$ and $\boldsymbol{b}$. This effect is well known in case of $\mathrm{Ti}$ 2p-absorption spectra of $\mathrm{TiO}_{2}$ and more expressed in the shape of feature $\boldsymbol{b}$ connected with the double-degenerate eg component [23, 30-35].

The analysis of the measured spectra of the samples of the first group (Figure 1c) reveals that in an

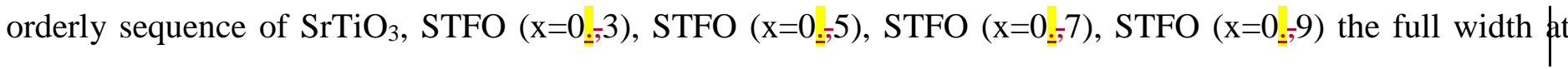
half maximum (FWHM) for peak $\boldsymbol{b}$ is equal to $0.8 \mathrm{eV}, 1.2 \mathrm{eV}, 1.3 \mathrm{eV}, 1.4 \mathrm{eV}$ and $1.5 \mathrm{eV}$, respectively. Hence, the FWHM value for peak $\boldsymbol{b}$ increases with increasing Fe content. The uncertainty in peak width is about $0.05 \mathrm{eV}$, and is specified by the accuracy determining the values of the FWHM and also by the accuracy of background subtraction for Ti L2,3-absorption spectra features. It is plausible to assume that the observed changes in feature $\boldsymbol{b}$ originate from distortions of the $\mathrm{TiO}_{6}$ octahedra in STFO occurred during the substitution of $\mathrm{Ti}$ atoms by Fe. We therefore assume that during the doping process the iron atoms replace the titanium atoms, thereby inducing structural distortions. 
As to the second group of STFO samples, the same tendencies can be traced. Additionally, it is noteworthy that the shape of $\mathrm{Ti} 2 \mathrm{p}_{3 / 2}$ absorption spectra of STFO samples with $\mathrm{x}=0.25$ and 0.35 content of the Fe are almost indistinguishable suggesting similar local structure around the Ti atoms for these two samples.

\subsection{Iron 2p X-ray absorption}

The Fe 2p ( $\left.\mathrm{L}_{2,3}\right)$-absorption spectra of STFO systems with a partial substitution of Ti by Fe atoms are presented in Figure 2. The Fe $\mathrm{L}_{2,3}$ - absorption spectrum of $\mathrm{SrFeO}_{3}$ taken from ref. [36] is also shown.
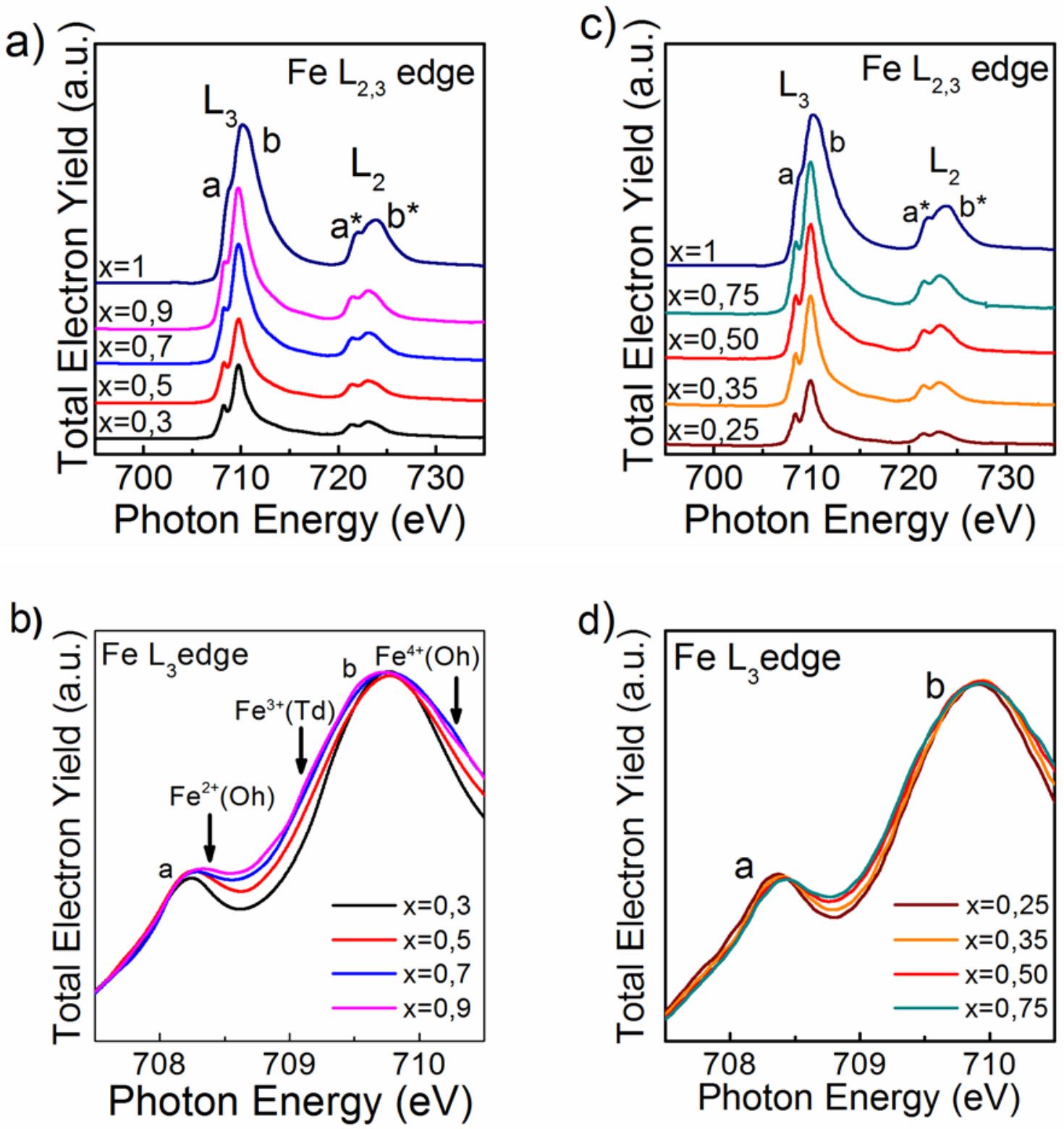

Figure 2. Fe 2p $\left(\mathrm{L}_{2,3}\right)$ - absorption spectra of the $\mathrm{SrTi}_{1-\mathrm{x}} \mathrm{Fe}_{\mathrm{x}} \mathrm{O}_{3-\delta}$ (STFO) samples with different content of Fe on B-site of perovskite structure: (a-b) a first group of samples with $\mathrm{x}$ : 0.3, 0.5, 0.7 and 0.9 of Fe atoms produced by spray pyrolysis method; (c-d) a second group of samples with x: $0.25,0.35,0.50$ and 0.75 of $\mathrm{Fe}$ 
atoms produced by a modified Pechini method. Panels (b) and (d) show the Fe 2p ( $\left.\mathrm{L}_{2,3}\right)$ - absorption spectra normalized on the intensity of the feature $\boldsymbol{b}$. The $\mathrm{Fe} \mathrm{L}_{2,3}-$ absorption spectrum of $\mathrm{SrFeO}_{3}$ is taken from ref. [36].

According to the classical conception, the NEXAFS excitation at the Fe 2p threshold should reflect the energies of the empty Fe $3 d$ states, because it is dominated by the $2 p \rightarrow 3 d$ dipole transition in Fe atoms [37].

One can see that all the studied Fe 2p absorption spectra clearly reflect the spin-orbit splitting of the Fe

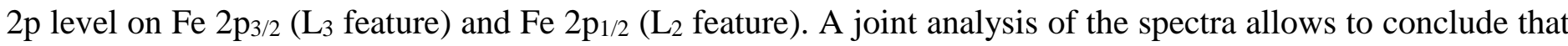
growth of Fe content in both groups of STFO samples leads to increase of the integral intensity of the $\mathrm{L}_{3}$ absorption band while maintaining the spin-orbit splitting. Noteworthy that unlike the spectrum of pristine structure $\mathrm{SrFeO}_{3}$, the double-peaked $\mathrm{Fe} \mathrm{L}_{3}$ absorption band is traced in all the spectra of Fe substituted STFO systems. Moreover, the contrast of feature $\boldsymbol{a}$ relative to feature $\boldsymbol{b}$ depends also on the Fe content in STFO.

To understand the nature of the observed evolution of the double-peaked Fe $\mathrm{L}_{3}$ absorption band let us turn to the analysis of Fe 2p absorption spectra of reference systems. The spectra for iron oxides characterized by different coordination sites and oxidation states for iron ions are shown in Figure 3. The Fe 2p -absorption spectra of $\alpha-\mathrm{Fe}_{2} \mathrm{O}_{3}$ and $\mathrm{Fe}_{3} \mathrm{O}_{4}$ powders were measured under the same experimental conditions as it was carried out for Fe substituted STFO systems. The Fe 2p-absorption spectra of $\mathrm{FeO}$ and $\mathrm{SrFeO}_{3}$ were taken from [36].

A joint analysis of the Fe 2p-absorption spectra for the referred structures reveals that the shape of the Fe $\mathrm{L}_{3}$ absorption band (the appearance of feature $\boldsymbol{a}$ ) depends strongly on the oxidation state and coordination number of Fe atoms. The observed tendency is due to the different correlation between the relative energy differences resulting from the ligand-field splitting (D) and exchange splitting (J) in different systems. The former is determined by the energy difference between the $2 t_{2 g}$ and $3 e_{g}$ orbitals. The latter is due to the exchange repulsion between the d-electrons in the pairwise configuration. Notice that due to a large number of unpaired spins a strong exchange splitting interaction $(\mathrm{D}<\mathrm{J})$, which splits the $3 \mathrm{t}_{2 \mathrm{~g}}$ and $2 \mathrm{e}_{\mathrm{g}}$ orbitals into four separate states occurs in all the above mentioned compounds. ${ }^{38}$

One can see that the greatest splitting of the Fe $\mathrm{L}_{3}$ absorption band on two features $\boldsymbol{a}$ and $\boldsymbol{b}$ occurs in Fe $2 p$ absorption spectrum of $\alpha-\mathrm{Fe}_{2} \mathrm{O}_{3} . \alpha-\mathrm{Fe}_{2} \mathrm{O}_{3}$ has a corundum type structure $\left(\alpha-\mathrm{Al}_{2} \mathrm{O}_{3}\right)$ where Fe ions occupy nearly perfect octahedral sites with formally $\mathrm{Fe}^{3+}$ oxidation state [39] that leads to formally $3 \mathrm{~d}^{5}$ electronic configuration of Fe ions, which corresponds to a half-filled d-subshell and is particularly most stable in highspin configuration [40].

As opposed to Fe 2p absorption spectrum of $\alpha-\mathrm{Fe}_{2} \mathrm{O}_{3}$ the presence of unsplit $\mathrm{Fe} \mathrm{L}_{3}$ absorption band (no splitting of the Fe $\mathrm{L}_{3}$ absorption band into features $\boldsymbol{a}$ and $\boldsymbol{b}$ ) in the spectra of $\mathrm{FeO}$ and $\mathrm{SrFeO}_{3}$ oxides (Figure 3) with the formal oxidation states $\mathrm{Fe}^{2+}\left(3 d^{6}\right)$ and $\mathrm{Fe}^{4+}\left(3 d^{4}\right)$, respectively [39], is traced. Analysis of the configuration energy difference, including the crystal field and charge transfer parameters, allowed the authors 
of $[39,41,42]$ to conclude that the observed difference is mostly due to the change in the number of occupations of $d$ orbitals in high spin configuration having formally [Ar] $3 d^{5}$, [Ar] $3 d^{6}$ and $[\mathrm{Ar}] 3 \mathrm{~d}^{4}$ ground-state electronic configurations for ions $\mathrm{Fe}^{3+}, \mathrm{Fe}^{2+}$ and $\mathrm{Fe}^{4+}$ in $\alpha-\mathrm{Fe}_{2} \mathrm{O}_{3}, \mathrm{FeO}, \mathrm{SrFeO}_{3}$, respectively. As compared with $\alpha-\mathrm{Fe}_{2} \mathrm{O}_{3}$, a larger value of the configuration energy difference (4.0 eV against $2.2 \mathrm{eV}$ in $\alpha-\mathrm{Fe}_{2} \mathrm{O}_{3}$ ) and a larger iron-oxygen distance is traced in $\mathrm{FeO}$ [39] that leads to less important charge transfers in this compound and can be seen in the smaller weight of the ligand hole $\left(d^{7} \underline{L}\right)$ configuration. $\underline{L}$ denotes a hole due to a charge transfer from ligand 2p to transition-metal 3d state.

According to [42, 43], large overlap between Fe $3 d$ and $\mathrm{O} 2 p$ states exists in $\mathrm{SrFeO}_{3}$, suggesting strong hybridization between these states. In stoichiometric $\mathrm{SrFeO}_{3}$ the formally tetravalent $\mathrm{Fe}^{4+}$ ions $\left(3 \mathrm{~d}^{4}\right)$ are in the high spin state, and they are successfully described by a sum $3 \mathrm{~d}^{5} \underline{\mathrm{L}}$ and $3 \mathrm{~d}^{4}$ electronic configurations. The occupation of the $3 d^{5} \underline{L}$ configuration is larger than that of the $3 d^{4}$ configuration and as a consequence, $\mathrm{SrFeO}_{3}$ is in the negative charge-transfer regime. This conclusion agrees well with conclusion made in 41 that in $\mathrm{SrFeO}_{3}$ the effective value of negative charge-transfer energy is $\Delta \sim-3 \mathrm{eV}$.

$\mathrm{Fe}_{3} \mathrm{O}_{4}$ has an inverse spinel structure [44], where Fe ions are in mixed oxidation state $\mathrm{Fe}^{2+} / \mathrm{Fe}^{3+}$ with a ratio of 0.5 . In each unit cell, the tetrahedral sites are occupied by $\mathrm{Fe}^{2+}$ ions while the octahedral sites are occupied by a random distribution of $\mathrm{Fe}^{2+}$ and $\mathrm{Fe}^{3+}$ ions. As follows from the calculations [39], the global spectrum of $\mathrm{Fe}_{3} \mathrm{O}_{4}$ is a summation of the three associated spectra.

Let us turn to Figure2 where the Fe 2p absorption spectra of STFO compounds with different Fe content are shown. As mentioned above, the $\mathrm{SrTi}_{1-\mathrm{x}} \mathrm{Fe}_{\mathrm{x}} \mathrm{O}_{3-\delta}$ sequence forms a continuous solid solution between end members $\mathrm{SrTiO}_{3}$ and $\mathrm{SrFeO}_{3}$. The joint analysis of the Fe2p- absorption spectra of all the studied STFO structures reveals significant differences from the Fe 2p-absorption spectrum of $\mathrm{SrFeO}_{3}$.

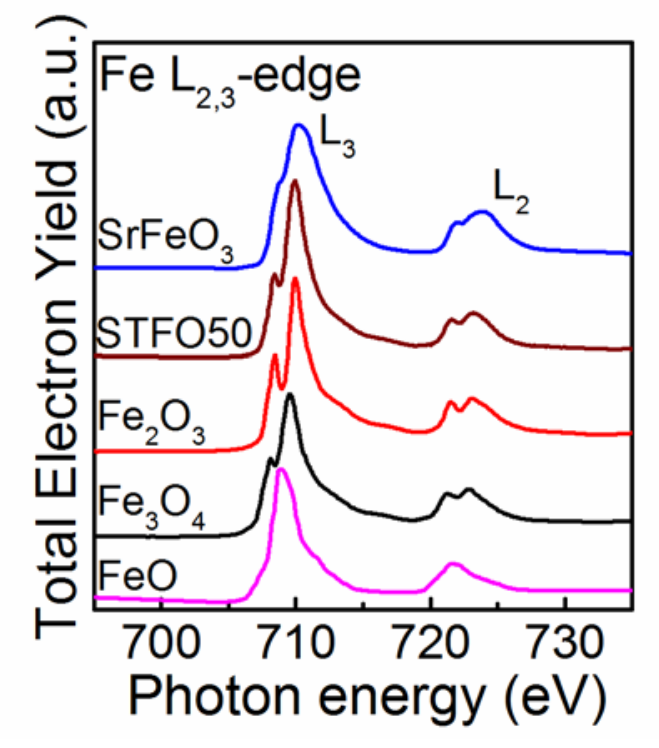


Figure 3. Fe2p ( $\left.\mathrm{L}_{2,3}\right)$ - absorption spectra of the reference structures $\mathrm{FeO}, \alpha-\mathrm{Fe}_{2} \mathrm{O}_{3}, \mathrm{Fe}_{3} \mathrm{O}_{4}, \mathrm{SrFeO}_{3}$ and $\mathrm{SrTi}_{1-\mathrm{x}} \mathrm{Fe}_{\mathrm{x}} \mathrm{O}_{3-\delta}$ with $\mathrm{x}=0.5$ (STFO50) sample produced by a modified Pechini method. The Fe $\mathrm{L}_{2,3}$-absorption spectra of $\mathrm{FeO}$ and $\mathrm{SrFeO}_{3}$ were taken from [36].

One can conclude that incorporation of $\mathrm{Fe}$ in $\mathrm{SrTiO}_{3}$ structure leads to the formation of Fe-sites with oxidation states and coordinations differing from $\mathrm{SrFeO}_{3}$. The evolution of the intensity and shape (contrast) of feature $\boldsymbol{a}$ relative to feature $\boldsymbol{b}$ also points to a change in the oxidation state or coordination of Fe ions in STFO with increasing Fe content. One can see that the most pronounced peak $\boldsymbol{a}$ exists in the STFO sample with the minimal Fe content (x=0.3) (Figure2a). The contrast of feature $\boldsymbol{a}$ decreases with growth of Fe concentration with respect to the contrast of feature $\boldsymbol{b}$. It should be also noted that the energy distance $\Delta \mathrm{E}$ between features $\boldsymbol{a}$ and $\boldsymbol{b}$ in Fe2p- absorption spectra is minimal in the STFO $(\mathrm{x}=0 .-9)$ and equals to1.38 eV that is close to the $\Delta \mathrm{E}$ value for $\mathrm{Fe}_{3} \mathrm{O}_{4}$. For all other concentrations the distance between features $\boldsymbol{a}$ and $\boldsymbol{b}$ is about $1.52 \mathrm{eV}$ that correlates well with the $\Delta \mathrm{E}$ value for $\alpha-\mathrm{Fe}_{2} \mathrm{O}_{3}$.

The joint analysis of feature $\boldsymbol{a}$ and $\triangle \mathrm{E}$ value for STFO compounds and reference systems reveals the following tendencies in Fe2p- absorption spectra of the STFO samples depending on Fe content. The Fe 2pabsorption spectrum of STFO $(x=0 .-3)$ is similar to that of $\alpha-\mathrm{Fe}_{2} \mathrm{O}_{3}$. That means that Fe ions in the STFO with $\mid \mathrm{a}$ small Fe concentration are preferentially in the $\mathrm{Fe}^{3+}$ oxidation state $\left(3 d^{5}\right)$ in octahedral coordination along with essentially smaller content of $\mathrm{Fe}^{4+}$ states in octahedral environment.

With increasing Fe content in Fe L3- absorption spectra of STFO there are:

1) gradual decrease in the contrast of feature $\boldsymbol{a}$ regarding to feature $\boldsymbol{b}$;

2) gradual broadening of feature $\boldsymbol{b}$;

3) broadening of feature $\boldsymbol{a}$ and a slight shift of feature $\boldsymbol{a}$ maximum towards higher energies.

The broadening of feature $\boldsymbol{b}$ in Fe $\mathrm{L}_{3}$ absorption band, which is accompanied by small shift to higher energy indicates the growth of the amount of $\mathrm{Fe}^{4+}$ ions in octahedral $(\mathrm{Oh})$ coordinations in STFO with increasing the $\mathrm{Fe}$ atoms content in the structure. However, the contribution of $\mathrm{Fe}^{4+}$ states, most likely, is not significant, because the overall shape of the Fe 2p absorption spectrum, the energy position and width of the Fe $\mathrm{L}_{3}$ absorption band for all the STFO samples are very different from the $\mathrm{Fe}_{3}$ absorption spectrum of $\mathrm{SrFeO}_{3}$ $\left(\mathrm{Fe}^{4+}\right)$.

According to the calculations [45, 46], the broadening of feature $\boldsymbol{b}$ in the Fe $\mathrm{L}_{3}$ absorption band toward lower energies is caused by an appearance of $\mathrm{Fe}^{3+}$ states in tetrahedral (Td) coordinations in STFO.

In addition, in the Fe 2p absorption spectra of STFO samples with Fe concentration $\geq 70 \%$ there is a broadening of feature $\boldsymbol{a}$, that indicates the appearance of some amount of $\mathrm{Fe}^{2+}$ ions in octahedral environment $[45,47]$. The effect is strongly expressed in the Fe 2p- absorption spectrum of STFO ( $x=0.99)$ sample which is 
very similar to $\mathrm{Fe} 2 \mathrm{p}$ absorption spectrum of $\mathrm{Fe}_{3} \mathrm{O}_{4}$. This indicates that besides $\mathrm{Fe}^{3+}$ (in octahedral Oh and tetrahedral $\mathrm{Td}$ coordinations) and $\mathrm{Fe}^{4+}$ (in octahedral Oh coordination) ions in STFO ( $\mathrm{x}=0 .{ }_{-5}$ ) structure the presence of $\mathrm{Fe}^{2+}$ ions in octahedral sites is most likely leading to broadening of feature $\boldsymbol{a}$ in the Fe 2p absorption spectrum, accompanied by a decrease in feature $\boldsymbol{a}$ contrast regarding to feature $\boldsymbol{b}$.

On the basis of the carried analysis of the Fe 2p absorption spectra of STFO compounds one can conclude that in all the studied STFO structures Fe ions are present predominantly in $\mathrm{Fe}^{3+}$ oxidation state in octahedral environment along with essentially smaller content of $\mathrm{Fe}^{4+}$ states in octahedral environment. However, with growth of Fe content in STFO ( $x=0.5-5,-7)$ the $\mathrm{Fe}^{4+}$ states in octahedral sites $(\mathrm{Oh})$ and $\mathrm{Fe}^{\beta+}$ states in tetrahedral sites (Td) occurs additionally to the main state ( $\mathrm{Fe}^{3+}$ states in octahedral site). Moreover, the STFO $(x=0, .99)$ structure contains most likely $\mathrm{Fe}^{2+}(\mathrm{Oh})$ ions besides the $\mathrm{Fe}^{3+}(\mathrm{Td}+\mathrm{Oh})$ and $\mathrm{Fe}^{4+}(\mathrm{Oh})$ ions.

The obtained result is in a good agreement with results reported in literature $[48,49]$ according to that Fe ions in STFO have mixture of $\mathrm{Fe}^{3+}(\mathrm{Oh})$ and $\mathrm{Fe}^{4+}(\mathrm{Oh})$ states. It should be also noted that according to [50] the Fe ions in STFO can have a mixed $\mathrm{Fe}^{2+/} \mathrm{Fe}^{3+}(\mathrm{Oh}+\mathrm{Td})$ oxidation state.

Concerning the second group of the STFO samples, it should be emphasized that in the spectra of these samples the dependence of the shape of peak $\boldsymbol{a}$ on the Fe content is almost absent. The slight differences in the Fe dependence of the two sets of STFO powders can probably be assigned to the different synthesis routes $\phi \mathrm{f}$ the powders. Due to the different calcination temperatures applied in the two synthesis routes, the concentration of oxygen in the two sets might be slightly different reflected in the absorption spectra of Fe.

Fe 2p- absorption spectra of STFO samples with 25\% and 35\% of Fe content look like quite similar. The energy distance $(\Delta \mathrm{E})$ between features $\boldsymbol{a}$ and $\boldsymbol{b}$ in Fe 2p absorption spectra of STFO $(\mathrm{x}=0 .-25)$ and STFO $(\mathrm{x}=0 .-35)$ samples correlates with $\Delta \mathrm{E}$ for $\alpha-\mathrm{Fe}_{2} \mathrm{O}_{3}$. One can assume that for these samples the $\mathrm{Fe}^{3+}$ states in octahedral sites (Oh) state is most typical.

The mean oxidation state of Fe depends on temperature. For all STF systems, the concentration $\phi \mathrm{f}$ oxygen vacancies is assumed to increase with increasing temperature thus enhancing the oxygen diffusivity. Gradual rise of charge compensation by increasing the concentration of $\mathrm{Fe}^{2+}$ and $\mathrm{Fe}^{3+}$ on the expense of $\mathrm{Fe}^{4+}$ $\left(3 \mathrm{~d}^{4}\right)$ and $\mathrm{Fe}^{3+}\left(3 \mathrm{~d}^{5} \mathrm{~L}\right)$ is thus expected with increasing temperature.

\subsection{Oxygen 1s X-ray absorption}

O1s (K)- absorption spectra of the $\mathrm{SrTi}_{1-\mathrm{x}} \mathrm{Fe}_{\mathrm{x}} \mathrm{O}_{3-\delta}(\mathrm{STFO})$ with different content of Fe atoms are shown in Figure4. OK- absorption spectra of $\mathrm{SrTiO}_{3}, \mathrm{SrFeO}_{3}$ and $\mathrm{SrO}$ are also shown. O K- absorption spectra of $\mathrm{SrTiO}_{3}$ and $\mathrm{SrO}$ were measured under the same experimental conditions. The $\mathrm{O} \mathrm{K}$-absorption spectrum of $\mathrm{SrFeO}_{3}$ was taken from [42]. 

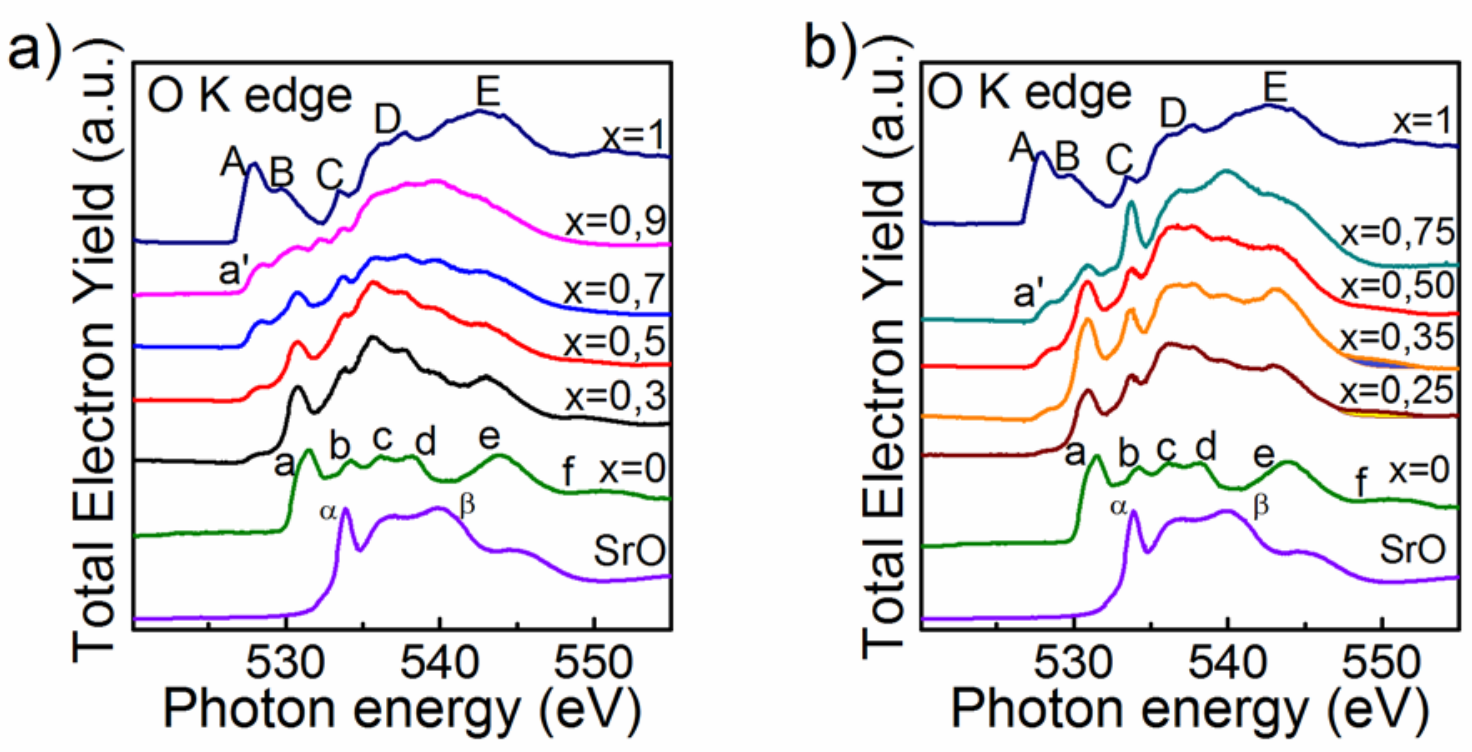

Figure 4. O1s (K)- absorption spectra of $\mathrm{SrTi}_{1-\mathrm{x}} \mathrm{Fe}_{\mathrm{x}} \mathrm{O}_{3-\delta}$ (STFO) with different Fe content: (a) a first group of samples with $\mathrm{x}$ : $0.3,0.5,0.7$ and 0.9 of Fe atoms produced by spray pyrolysis method; (b) a second group of samples with $\mathrm{x}$ : $0.25,0.35,0.50$ and 0.75 of Fe atoms produced by a modified Pechini method. OKabsorption spectra of $\mathrm{SrTiO}_{3}, \mathrm{SrFeO}_{3}$ and $\mathrm{SrO}$ are also shown. O K- absorption spectra of $\mathrm{SrTiO}_{3}$ and $\mathrm{SrO}$ were measured under the same experimental conditions. The $\mathrm{O} \mathrm{K}$-absorption spectra of $\mathrm{SrFeO}_{3}$ was taken from [42].

Let us firstly discuss the formation of the OK-absorption spectrum of $\mathrm{SrTiO}_{3}$. Because the $\mathrm{SrTiO}_{3}$ can be described by stacking of $\mathrm{TiO}_{2}$ and $\mathrm{SrO}$ layers, the $\mathrm{O} 1 \mathrm{~s}$ absorption spectrum of this structure is a superposition of the $\mathrm{TiO}_{2}$ and $\mathrm{SrO}$ spectra displaying separately each structure. The analysis of energy position of $\mathrm{O} 1$ s-absorption spectra of $\mathrm{TiO}_{2}[23,30,34,51]$ and $\mathrm{SrO}$ [52] indicates that these spectra differ significantly from each other in the energy positions of the main spectral details that allow their separate analysis.

$\mathrm{O}$ 1s-absorption spectrum of $\mathrm{TiO}_{2}$ originates mainly from $\mathrm{O}$ 1s-to-valence transitions located below and above the threshold. The covalent bonding of the Ti $3 d$ with the $\mathrm{O} 2 \mathrm{p}$ states forms the unoccupied $t_{2 g}$ and $e_{g}$ orbitals in the octahedral field. In $\mathrm{SrTiO}_{3}$ the band corresponding to the $\sigma$ bonding of $\mathrm{Ti} 3 \mathrm{~d}$ and $\mathrm{O} 2 \mathrm{p}$ atomic orbitals ( $e_{g}$ component) is rather broad and is reflected in the $\mathrm{O}$ 1s-edge absorption spectrum as the peak $\boldsymbol{b}$. In contrast, the weak $\pi$ bonding of the Ti $3 \mathrm{~d}$ and the $\mathrm{O} 2 \mathrm{p}$ orbitals ( $\mathrm{t}_{2 \mathrm{~g}}$ component) results in a narrow and sharp peak $\boldsymbol{a}$. According to [52] the features $\boldsymbol{c}, \boldsymbol{d}$, and $\boldsymbol{e}$ are dominated by strontium p-character mixing with $\mathrm{O} 2 \mathrm{p}$ states and the wide $\boldsymbol{f}$ band traced in between $547 \mathrm{eV}$ and $553 \mathrm{eV}$ can be assigned to titanium 4 sp band mixing with $\mathrm{O} 2 \mathrm{p}$ states. The strontium related feature $\boldsymbol{c}$ is slightly overlapping with $\mathrm{Ti} 3 \mathrm{~d} \rightarrow \mathrm{e}_{\mathrm{g}}$ feature. Note that in octahedral complexes the metal $e_{g}$ orbitals are directed towards the oxygen atoms and have a stronger overlap 
with the $\mathrm{O} 2 \mathrm{p}$ orbitals. As a result, the $\mathrm{O}$ 1s-near edge structure is very sensitive to changes in the local $\mathrm{Ti}$ geometry.

As follows from Figure 4, the O 1s-absorption spectra of STFO with Fe content of 30\%, 50\% and 70\% are similar to that of $\mathrm{SrTiO}_{3}$. With increasing the iron content: i) the spectra in general maintain the number of main features and their energy position; ii) contrast of all the features of $\mathrm{O}$ 1s-absorption spectra of STFO decreases; iii) an additional feature $\boldsymbol{a}^{\prime}$ exists in all the spectra of STFO as opposed to $\mathrm{SrTiO}_{3}$. Its intensity increases with growth of Fe content. Following the established tendencies it is reasonable to expect that the feature $\boldsymbol{a}^{\prime}$ originates from the Fe states in the structure STO. It is important that the energy position of feature $\boldsymbol{a}^{\prime}$ does_no-t depend on Fe content in STFO.

Let us shortly turn to the OK-absorption spectrum of $\mathrm{SrFeO}_{3}$ (Figure 4). This spectrum corresponds to transitions from the $\mathrm{O} 1 s$ to unoccupied $\mathrm{O} 2 p$ states in the conduction band. Due to strong oxygen-metal hybridization the first double feature A-B has a mostly metal character and originates from bonding of $\mathrm{O} 2 \mathrm{p}$ states with Fe 3d states forming the unoccupied $t_{2 g}$ and $e_{g}$ orbitals in the octahedral field. The rising structure C$\mathrm{D}$ in $\mathrm{O}$ 1s-absorption spectrum of SFO in the vicinity of $535 \mathrm{eV}$ corresponds to the $\mathrm{Sr} 4 d$ band region, and the structure $\mathrm{E}$ in the region $540-545 \mathrm{eV}$ originates predominantly from the Fe 4sp states [42]. Comparison of energy positions of feature $\boldsymbol{a}^{\prime}$ in O 1s-absorption spectra of STFO and the feature $\boldsymbol{A}$ in the spectrum of $\mathrm{SrFeO}_{3}$ reveals a small shift of feature $\boldsymbol{a}^{\prime}$ towards higher energies with respect to $\boldsymbol{A}$, which indicates a different oxidation state of Fe ions in STFO compounds from $\mathrm{SrFeO}_{3}$. Such conclusion agrees well with result obtain by analysis of $\mathrm{Fe} \mathrm{L} 2,3$-absorption spectra.

It should be also noted that according to [30, 53-55], in the pre-edge region of OK-absorption spectrum of non-stoichiometric $\mathrm{SrTiO}_{3}$ there is a feature connected with oxygen defect states. The intensity of this detail depends strongly on the concentration of oxygen vacancies in $\mathrm{SrTiO}_{\mathrm{x}}$. The energy position of this peak coincides with position of feature $\boldsymbol{a}^{\prime}$ in O 1s-absorption spectra of STFO. This means that feature $\boldsymbol{a}^{\prime}$ reflects both the presence of Fe atoms and oxygen vacancies states in STFO.

Let us turn to the O 1s-absorption spectra of the second group STFO. The main trends established for first group can be also traced here. At the same time, the $\mathrm{O}$ 1s-absorption spectrum of the STFO $(\mathrm{x}=0 .-35)$ sample drops out from the common sequence. This spectrum is allocated by manifestation of its main details, especially $\boldsymbol{b}$ and $\boldsymbol{e}$. According to [30] the sharp intensive features $\boldsymbol{b}$ and $\boldsymbol{e}$ correspond to the ideal cubic $\mathrm{SrTiO}_{3}$ structure. Also the feature $\boldsymbol{f}$ is to some extent the indicator of the stoichiometric structure with signs of crystallinity. The absence of this feature points to the violation of the STO structure. One can presume that the structure of STFO $\left(x_{-0}=35\right)$ sample is most closely matched to the perovskite cubic phase. Analysis of FeL2,3 - absorption spectra of STFO indicates that for this sample the high content of $\mathrm{Fe}^{3+}$ ( $3 \mathrm{~d}^{5}$ electronic configuration) ions is typical. Due to charge compensation, a high content of $\mathrm{Fe}^{3+}$ ions provides a large number of oxygen vacancies in the STFO structure. As oxygen diffusions proceeds via oxygen vacancies, a high concentration of oxygen vacancies supports diffusivity. On the other hand, a large vacancy concentration might lead to structural instability during longer exposure at high temperatures. The composition range of $25-25 \%$ 
Fe where local distortions are small seems, therefore, to be ideal with respect to stability and diffusivity.

Thus, it is plausible to presume that the substitution of Ti atoms in STO by $25 \%-35 \%$ of Fe atoms creates the optimum conditions for high electronic and ionic conductivity in the STFO, maintaining the cubic perovskite phase. It should be mentioned here that Schulze-Kueppers et al. [21] found 25 - 35 \% Fe substituting Ti seems to be a good compromise between structural and functional properties of STFO.

A particular attention is also attracted to a high intensity narrow feature $\boldsymbol{B}$ in the $\mathrm{O}$ 1s-absorption spectrum of STFO ( $\left.\mathrm{x}=0_{-,}, 75\right)$. A joint analysis of this spectrum and the spectrum of SrO showed that the energy position of feature $\boldsymbol{\alpha}$ (this feature originates from the $\mathrm{Sr} 4 \mathrm{~d}-\mathrm{O} 2 \mathrm{p}$ hybridization in $\mathrm{SrO}$ ) in the spectrum of the $\mathrm{SrO}$ is close to the energy position of feature $\boldsymbol{b}$ ( $\mathrm{e}_{\mathrm{g}}$ - component) in the O 1s-absorption spectra of STFO. Moreover, in this energy region the feature $\mathrm{C}$ originated from mixed $\mathrm{O} 2 \mathrm{p}$ and $\mathrm{Sr} 4 \mathrm{~d}$ states in $\mathrm{SrFeO}_{3}$ is located. One can presume that presence of the high intensity feature $\boldsymbol{B}$ in the $\mathrm{O}$ 1s-absorption spectra of STFO (especially in the sample STFO $\left(\mathrm{x}_{-5}=0.75\right)$ ) is associated with the formation of a large number of Sr-O bonds in these compounds. Thereby, it is plausible to assume that there are SrO precipitates in the STFO $\left(\mathrm{x}_{-1}=0 .-7 \$\right)$ sample. The segregation of Sr-rich layers at the surface of complex perovskites is a well-known phenomenon largely investigated in the course of solid oxide fuel/electrolysis cells (SOF/EC) cathodes [Yildiz 2012]. The segregation is observed in any kind of Sr-containing perovskites such as (La,Sr)-manganites or -cobaltites [Lee2013] as well as strontium titanates [Chen2012, Ohsawa2014]. Its occurrence is highly accepted and its impact on functional properties such as catalytic activity towards oxygen reduction is still under investigation. The exact mechanisms, however, are not totally understood and might differ for different compositions and conditions. The formation of $\mathrm{SrO}_{*}$ inside $\mathrm{SrTiO}_{3}$ is well known and is associated with the presence of a large number of defects in the $\mathrm{SrTiO}_{3}$ structure [56]. One can conclude that in samples prepared by the modified Pechini synthesis method the tendency of the formation of $\mathrm{SrO}_{x}$ is traced.

\section{Conclusions}

Two series of $\mathrm{SrTi}_{1-\mathrm{x}} \mathrm{Fe}_{\mathrm{x}} \mathrm{O}_{3-\delta}$ (STFO) powders with different Fe content produced by two different methods, solid state reaction or modified Pechini synthesis, have been investigated by soft X-ray absorption spectroscopy. The O1s (K)- , Fe2p ( $\left.\mathrm{L}_{2,3}\right)$ - and Ti2p $\left(\mathrm{L}_{2,3}\right)$ - absorption spectra of STFO powders were analyzed. Joint analysis of the Ti2p ( $\left.\mathrm{L}_{2,3}\right)$-absorption spectra of STFO structures testified that partial substitution of Ti by Fe atoms in $\mathrm{SrTiO}_{3}$ hint to asymmetric distortion of $\mathrm{TiO}_{6}$ octahedrons, which may violate the cubic symmetry of STFO. It was established that the distortion of $\mathrm{TiO}_{6}$ octahedrons are minimal in STFO samples with 25\%35\% Fe content and increases with increasing Fe content.

Analysis of the Fe2p $\left(\mathrm{L}_{2,3}\right)$-absorption spectra reveals that the main state of Fe ions for all STFO compounds is $\mathrm{Fe}^{3+}$ in an octahedral environment along with $\mathrm{Fe}^{4+}$ states in an octahedral environment where the latter 
contribution increases with increasing Fe content. The joint analysis of the spectra of STFO with reference compounds points also to the presence of $\mathrm{Fe}^{3+}$ states in a tetrahedral environment at high content of $\mathrm{Fe}$ atoms and a certain amount of $\mathrm{Fe}^{2+}$ ions in an octahedral environment in the STFO compound with Fe content more than $75 \%$.

Analysis of the pre-edge region of the O1s (K)- absorption spectra reveals a specific feature which is related with formation of oxygen vacancies in STFO. Moreover, the intensity of these features increase with increasing Fe content. The lowest degree of structure distortions traced in STFO $(x=0.35)$. Hence, the STFO $\left(x_{=0}=0.3 \$\right)$ compound seems to be mostly appropriate for technical applications in agreement with literature [21].

Additionally, a careful analysis of all the spectra reveals that the modified Pechini method allows to synthesize more stable structures but a tendency of the $\mathrm{SrO}_{x}$ formation in the structure in this method was marked. The spray pyrolysis method gives the structure free of SrO precipitates.

\section{Acknowledgments}

The authors acknowledge financial support from the German-Russian Interdisciplinary Science Center (G-RISC) funded by the German Federal Foreign Office via the German Academic Exchange Service (DAAD) (projects P-2013a-8, P-2014a-11). We thank HZB for the allocation of synchrotron radiation beamtime. Support from Helmholtz-Association through the Helmholtz-Alliance MEM-BRAIN is also gratefully acknowledged.

\section{References}

[1] A.J. Jacobson, Materials for Solid Oxide Fuel Cells, Chem. Mater. 22 (2010) 660-674.

[2] S.P. Jiang, Development of lanthanum strontium manganite perovskite cathode materials of solid oxide fuel cells: a review, J. Mater. Sci. 43 (2008) 6799-6838.

[3] Y. Li, R. Gemmen, X. Liu, Oxygen reduction and transportation mechanisms in solid oxide fuel cell cathodes, J. Power Sources 195 (2010) 3345-3358.

[4] C. Sun, R. Hui, J. Roller, Cathode materials for solid oxide fuel cells: a review, J. Solid State Electrochem. 14 (2010) 1125-1144.

[5] G.M. Choi, H.L. Tuller, D. Goldschmidt, Electronic-transport behavior in single-crystalline Ba0.0Sr0.97TiO3, Phys. Rev. B 34 (1986) 6972-6979.

[6] R.Meyer, R.Waser, Resistive donor-doped SrTiO3 sensors: I, basic model for a fast sensor response, Sens. Actuators B 101 (2004) 335-345. 
[7] W. Menesklou, H.-J. Schreiner, K.H. Härdtl, E. Ivers-Tiffée, High temperature oxygen sensors based on doped SrTiO3, Sens. Actuators B 59 (1999) 184-189.

[8] P. Meuffels, Propane gas sensing with high-density $\operatorname{SrTi0.6Fe} 0.4 \mathrm{O}(3-\delta)$ ceramics evaluated by thermogravimetric analysis, J. Eur. Ceram. Soc. 27 (2007) 285-290.

[9] G. Neri, G. Micali, A. Bonavita, R. Licheri, R. Orrù, G. Cao, D. Marzorati, E. Merlone Borla, E. Roncari, A. Sanson, FeSrTiO3-based resistive oxygen sensors for application in diesel engines Sens. Actuators B 134 (2008) 647-653.

[10] G. Jin, G. Choi, W. Lee, J. Park, Gas sensing property of perovskite SrTi1-xFexO3- $\delta$ fabricated by thick film planar technology, J. Nanosci. Nanotechnol. 11 (2011) 1738-1741.

[11] H.-S. Kim, L. Bi, H. Paik, D.-J. Yang, Y. C. Park, G. F. Dionne and C. A. Ross, Self-assembled single-phase perovskite nanocomposite thin films, Nano Lett. 10 (2010) 597-602.

[12] S. Molin, W. Lewandowska-Iwaniak, B. Kusz, M. Gazda and P. Jasinski, Structural and electrical properties of $\operatorname{Sr}(\mathrm{Ti}, \mathrm{Fe}) \mathrm{O}-\delta$ materials for SOFC cathodes, J. Electroceram. 28 (2012) 80-87.

[13] J. R. Jurado, F. M. Figueiredo, B. Gharbage, J. R. Frade, Electrochemical permeability of Sr0.7(Ti,Fe)O3- $\delta$ materials, Solid State Ionics 118 (1999) 89-97.

[14] J. Mizusaki, M. Okayasu, S. Yamauchi, K. Fueki, Nonstoichiometry and phase relationship of the SrFeO2.5-SrFeO3 system at high temperature, J. Solid State Chem. 99 (1992) 166-172.

[15] S. Steinsvik, R. Bugge, J. Gjønnes, J. Taftø, T. Norby, The defect structure of SrTi1-xFexO3-y $(\mathrm{x}=0-0.8)$ investigated by electrical conductivity measurements and electron energy loss spectroscopy (EELS), J. Phys. Chem. Solids 58 (1997) 969-976.

[16] A. Rothschild, W. Menesklou, H. L. Tuller and E. Ivers-Tiffée, Electronic structure, defect chemistry, and transport properties of SrTi1-xFexO3-y solid solutions, Chem. Mater. 18 (2006) 36513659.

[17] W. Jung, H. L. Tuller, Investigation of cathode behavior of model thin-film SrTi1-xFexO3$\delta(\mathrm{x}=0.35$ and 0.5$)$ mixed ionic-electronic conducting electrodes, J. Electrochem. Soc. 155 (2008) B1194-B1201.

[18] W. Jung, H. L. Tuller, Impedance study of SrTi1-xFexO3- $\delta(x=0.05$ to 0.80$)$ mixed ionicelectronic conducting model cathode, Solid State Ionics 180 (2009) 843-847. 
[19] W. Jung and H. L. Tuller, A new model describing solid oxide fuel cell cathode kinetics: model thin film SrTi1-xFexO3- $\delta$ mixed conducting oxides-a case study, Adv. Energy Mater. 1 (2011) 11841191.

[20] C.-Y. Yoo, Henny J. M. Bouwmeester, Oxygen surface exchange kinetics of SrTi1-xFexO3- $\delta$ mixed conducting oxides, Phys. Chem. Chem. Phys. 14 (2012) 11759-11765.

[21] F. Schulze-Küppers, S.F.P. ten Donkelaar, S. Baumann, P. Prigorodov, Y.J. Sohn, H.J.M. Bouwmeester, W.A. Meutenberg, O. Guillon, Structural and functional properties of SrTi1-xFexO3- $\delta$ $(0 \geq \mathrm{x} \geq 1)$ for the use as oxygen transport membrane, Sep. Purif. Technol. 147 (2015) 414-421.

[22] S.I. Fedoseenko, I.E. Iossifov, S.A. Gorovikov, J.-H. Schmidt, R. Follath, S.L. Molodtsov, V.K. Adamchuk, G. Kaindl, Development and present status of the Russian-German soft X-ray beamline at BESSY II, Nucl. Instrum. Methods Phys. Res., Sect. A 470 (2001) 84-88.

[23] F. M. F. de Groot, J. Faber, J. J. M. Michiels, M. T. Czyżyk, M. Abbate, J. C. Fuggle, Oxygen 1s x-ray absorption of tetravalent titanium oxides: A comparison with single-particle calculations, Phys. Rev. B 48 (1993) 2074-2080.

[24] M. Cardona, Optical properties and band structure of SrTiO3 and BaTiO3, Phys. Rev. 140 (1965) A651-A655.

[25] L. F. Mattheiss, Energy bands for KNiF3, SrTiO3, KMoO3, and KTaO3, Phys. Rev. B 6 (1972) 4718-4740.

[26] T. F. Soules, E. J. Kelly, D. M. Vaught, J. W. Richardson, Energy-band structure of SrTiO3 from a self-consistent-field tight-binding calculation, Phys. Rev. B 6 (1972) 1519-1532.

[27] P. Pertosa, F. M. Michel-Calendini, X-ray photoelectron spectra, theoretical band structures, and densities of states for BaTiO3 and KNbO3, Phys. Rev. B 17 (1978) 2011-2020.

[28] V.I. Grebennikov, V.R. Galakhov, L.D. Finkel'shtein, N.A. Ovechkina, É.Z. Kurmaev, Effect of atomic magnetic moments on the relative intensity of the $L \beta$ and $L \alpha$ components in $x$-ray emission spectra of 3d transition metal oxides, Phys. Solid State 45 (2003) 1048-1055.

[29] F. M. F. de Groot, M. O. Figueiredo, M. J. Basto, M. Abbate, H. Petersen, J. C. Fuggle, 2p X-ray absorption of titanium in minerals, Phys. Chem. Miner. 19 (1992) 140-147. 
[30] E.O. Filatova, A.A. Sokolov, Yu.V. Egorova, A.S. Konashuk, O.Yu. Vilkov, M. Gorgoi, A.A. Pavlychev, X-ray spectroscopic study of SrTiOx films with different interlayers, J. Appl. Phys. 113 (2013) 224301.

[31] E. Filatova, E. Taracheva, G. Shevchenko, A. Sokolov, I. Kozhevnikov, S. Yulin, F. Schaefers, W. Braun, Atomic ordering in TiO2 thin films studied by X-ray reflection spectroscopy, Phys. Status Solidi B 246 (2009) 1454-1458.

[32] R. Brydson, H. Sauer, W. Engel, J. M. Thomas, E. Zeitler, N. Kosugi, H. Kuroda, Electron energy loss and X-ray absorption spectroscopy of rutile and anatase: a test of structural sensitivity, J. Phys.: Condens. Matter 1 (1989) 797-812.

[33] J. P. Crocombette, F. Jollet, Ti 2p X-ray absorption in titanium dioxides (TiO2): the influence of the cation site environment, J. Phys.: Condens. Matter 6 (1994) 10811-10821.

[34] R. Ruus, A. Kikas, A. Saar, A. Ausmees, E. Nõmmiste, J. Aarik, A. Aidla, T. Uustare, I. Martinson, Ti 2p and O 1s X-ray absorption of TiO2 polymorphs, Solid State Commun. 104 (1997) 199-203.

[35] S. Räth, F. Gracia, F. Yubero, J. P. Holgado, A. I. Martin, D. Batchelor, A. R. González-Elipe, Angle dependence of the $\mathrm{O} \mathrm{K}$ edge absorption spectra of $\mathrm{TiO} 2$ thin films with preferential texture, Nucl. Instrum. Methods Phys. Res., Sect. B 200 (2003) 248-254.

[36] H. Ikeno, I. Tanaka, T. Miyamae, T. Mishima, H. Adachi, K. Ogasawara, First principles calculation of Fe L2,3-edge X-ray absorption near edge structures of iron oxides, Mater. Trans. 45 (2004) 1414-1418.

[37] B.K. Teo, P. A. Lee, Ab initio calculations of amplitude and phase functions for extended x-ray absorption fine structure spectroscopy, J. Am. Chem. Soc. 101 (1979) 2815-2832.

[38] J.G. Chen, NEXAFS investigations of transition metal oxides, nitrides, carbides, sulfides and other interstitial compounds, Surf. Sci. Rep. 30 (1997) 1-152.

[39] J.P. Crocombette, M. Pollak, F. Jollet, N. Thromat, M. Gautier-Soyer, X-ray-absorption spectroscopy at the Fe L2,3 threshold in iron oxides, Phys. Rev. B 52 (1995) 3143-3150.

[40] R.M. Cornell, U. Schwertmann, The Iron Oxides: Structure, Properties, Reactions, Occurrences and Uses; WILEY-VCH Verlag GmbH \& Co. KGaA: Weinheim, Germany, 2003. 
[41] A. E. Bocquet, A. Fujimori, T. Mizokawa, T. Saitoh, H. Namatame, S. Suga, N. Kimizuka, Y. Takeda, M. Takano, Electronic structure of SrFe4+O3 and related Fe perovskite oxides, Phys. Rev. B 45 (1992) 1561-1570.

[42] M. Abbate, G. Zampieri, J. Okamoto, A. Fujimori, S. Kawasaki, M. Takano, X-ray absorption of the negative charge-transfer material SrFe1-xCoxO3, Phys. Rev. B 65 (2002) 165120.

[43] M. Abbate, F.M.F. de Groot, J.C. Fuggle, A. Fujimori, O. Strebel, F. Lopez, M. Domke, G. Kaindl, G.A. Sawatzky, M. Takano, Y. Takeda, H. Eisaki, S. Uchida, Controlled-valence properties of La1-xSrxFeO3 and La1-xSrxMnO3 studied by soft-x-ray absorption spectroscopy, Phys. Rev. B 46 (1992) 4511-4519.

[44] M.E. Fleet, The structure of magnetite, Acta Crystallogr., Sect. B: Struct. Sci., Criyst. Eng. Mater. 37 (1981) 917-920.

[45] S.J. Brotton, R. Shapiro, G. van der Laan, J. Guo, P.-A. Glans, J.M. Ajello, Valence state fossils in Proterozoic stromatolites by L-edge X-ray absorption spectroscopy J. Geophys. Res.: Biogeosci. 112 (2007) G03004.

[46] D. Peak, T.Z. Regier, Direct observation of tetrahedrally coordinated Fe(III) in ferrihydrite, Environ. Sci. Technol. 46 (2012) 11473-11474.

[47] V.V. Mesilov, V.R. Galakhov, B.A. Gizhevskii, N.I. Lobachevskaya, M. Raekers, C. Taubitz, A.R. Cioroianu, M. Neumann, Valence states of iron ions in nanostructured yttrium iron garnet Y3Fe5O12 studied by means of soft X-ray absorption spectroscopy, J. Electron Spectrosc. Relat. Phenom. 185 (2012) 598-601.

[48] M. Ghaffari, T. Liu, H. Huang, O.K. Tan, M. Shannon, Investigation of local structure effect and X-ray absorption characteristics (EXAFS) of Fe (Ti) K-edge on photocatalyst properties of SrTi(1x)FexO(3- $\delta)$, Mater. Chem. Phys. 136 (2012) 347-357.

[49] M. Vračar, A. Kuzmin, R. Merkle, J. Purans, E. A. Kotomin, J. Maier, O. Mathon, Jahn-Teller distortion around Fe4+ in SrFexTi1-xO(3- $\delta$ ) from x-ray absorption spectroscopy, $\mathrm{x}$-ray diffraction, and vibrational spectroscopy, Phys. Rev. B 76 (2007) 174107.

[50] L.F. da Silva, W. Avansi Jr., M. L. Moreira, J. Andrés, E. Longo, V.R. Mastelaro, Novel SrTi1-xFexO3 nanocubes synthesized by microwave-assisted hydrothermal method, CrystEngComm. 14 (2012) 4068-4073. 
[51] F.M.F. de Groot, J.C. Fuggle, B.T. Thole, G.A. Sawatzky, L2,3 x-ray-absorption edges of d0 compounds: $\mathrm{K}+, \mathrm{Ca} 2+, \mathrm{Sc} 3+$, and Ti4+ in Oh (octahedral) symmetry, Phys. Rev. B 41 (1990) 928-937.

[52] J.L. Mansot, V. Golabkan, L. Romana, Ph. Bilas, E. Alleman, Y.Bercion, Tribological and physicochemical characterization of strontium colloidal additives in mild wear regime, Colloids Surf. A 243 (2004) 67-77.

[53] C. Århammar, A. Pietzsch, N. Bock, E. Holmström, C.M. Araujo, J. Gråsjö, S. Zhao, S. Green, T. Peery, F. Hennies, S. Amerioun, A. Föhlisch, J. Schlappa, T. Schmitt, V.N. Strocov, G.A. Niklasson, D.C. Wallace, J.-E. Rubensson, B. Johansson, R. Ahuja, Unveiling the complex electronic structure of amorphous metal oxides, Proc. Natl. Acad. Sci. U.S.A. 108 (2011) 6355-6360.

[54] G. Lucovsky, K.-B. Chung, J.-W. Kim, D. Norlund, Spectroscopic differentiation between Oatom vacancy and divacancy defects, respectively, in TiO2 and HfO2 by X-ray absorption spectroscopy, Microelectron. Eng. 86 (2009) 1676-1679.

[55] G. Lucovsky, L. Miotti, K. Paz Bastos, Many-Electron Multiplet Theory Applied to O-Atom Vacancies in High-к Dielectrics, Jpn. J. Appl. Phys. 50 (2011) 04DA15.

[56] T. Ohsawa, R. Shimizu, K. Iwaya, T. Hitosugi, Visualizing Atomistic Formation Process of SrOx Thin Films on SrTiO3, ACS Nano 8 (2014) 2223-2229. 


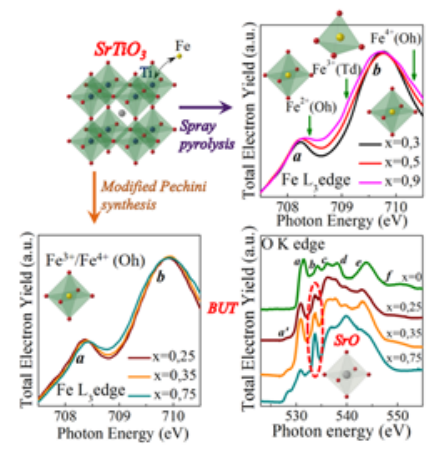

TOC Image 